\title{
Restraint Method Research for Coupling Random Error Based on High Dimensional Data Set Multiscale Analysis
}

\author{
X.Y. Zhou, J.Q. Wang, Z.M. Wang, Y.Y. Jiao X.G. \\ Pan \\ College of Science \\ National University of Defence Technology \\ Changsha, Hunan, China
}

\author{
C.E. Xiao \\ Hunan City University \\ Yiyang, Hunan, China
}

\begin{abstract}
For coupling random error, a new method based on multiscale analysis on high dimensional data set is advanced in this paper. It is extends traditional wavelet to high dimensional data set and does multiscale analysis, so that the information in the multi-measure data can be used better. The step of the restraint algorithm is given. Finally, simulated ship attitude data is used to verify the new method. The results show that the three angles in ship attitude are coupling and the method proposed in this paper is valid, which can get a better restraining result than traditional wavelet method.
\end{abstract}

Keywords-coupling error; restraint; high dimensional data set; multiscale; ship attitude

\section{INTRODUCTION}

Measurement error is inevitable because of measurement environment, measurement equipment and other factors. According to the mathematical processing treatments and their effects, measurement error can be divided into three classes: gross error, system error and random error.

Firstly, the gross error, which obviously makes measurement data deviated from the true value, will influence the measurement accuracy significantly. Therefore, identification methods are usually used to eliminate and reconstruct this kind of error $[1,2]$. Secondly, the system error, which has apparently regularity, will bring systematic bias in measurement data. So modeling system error based on measurement principle analysis is commonly employed to estimate and compensate it [3]. Thirdly, the random error, which has specific statistical character, will influence the stability of the measurement data. At present, the processing of the random error mainly has two aspects: one is statistical parameter estimation method, and the other one is smoothing method. For the first aspect, characteristic of the random error is tested at first, such as normality, independence and stationary, etc. If the random error is uncorrelated and smooth, lots of smoothing methods can be used. If the random error is correlative and smooth, time series modeling methods can be considered, such as AR (Auto-Regressive), MA (Moving Average) and ARMA (Auto-Regressive and Moving Average Model) etc. If the random error is nonstationary, time series modeling methods can be used [3]. For the latter aspect, there are several smoothing methods like spline based smoothing method, spline based signals separated method, wavelets $[4,5$,
6], EMD (Empirical Mode Decomposition)[7, 8, 9], time series analysis etc.

In fact, coupling is another important character for measurement data. For example, the continuous wave radar's measurement data of main and vice station, the GPS (Global Positioning System)/BDS (BeiDou Navigation Satellite System)differential data, ship attitude data, which expressed by correlated three direction attitude parameters, etc.. However, the mentioned methods cannot process coupling data efficiently That's because the relationship between multi-measurement data may be ignored by the traditional methods, which will induce information missing and influence the performance of data processing. Therefore, the purpose of this paper is to propose a new method, which is suitable to deal with coupling random error, based on analyzing and modeling the random error. The mainly approach we employed in this paper is to extend the traditional wavelet analysis to high dimensional set and does multiscale analysis to get the real measurement signal.

The structure of the paper is as follows. Following the introduction, the theory about multiscale analysis on high dimensional data set is presented. The application of multiscale analysis to suppress the coupling error of ship attitude data is depicted in Section 3. In section 4, simulations are used to validate the proposed method and the error-smooth effects are compared in the simulation tests under different methods. Finally, the paper is concluded in section 5.

\section{Multiscale Analysis On High Dimensional Data SET}

The multiscale analysis on high dimensional data set like the promotion of the wavelet analysis on it. But, strictly, it is not traditional wavelet analysis. The wavelet coefficients, which created by this transformation, satisfy the traditional relationship in position and scale. But the numerical value is difference, when these wavelet coefficients are matrix instead of real number. And this method is called the multiscale analysis on the high dimensional data set because the transformation is same as the traditional wavelet transformation.

Notes that the multiscale analysis used in this paper need the transform mapping in some point's tangent space in differential geometry, that is Exp and Log [10]. To standard 
high dimensional data set, the Exp and Log mapping can calculate directly by existing algorithm in it.

Examples will be provided below.

\section{SUPPRESSING OF SHIP ATTITUDE COUPLING ERROR}

In the above section, we introduce the principle of multiscale analysis on high dimensional data set. In this section, we takes the ship attitude measurement data as an example, and apply the novel method for coupling random error based on multiscale analysis on high dimensional data set.

\section{A. Coupling Random Error in Ship Attitude}

Marine instrumentation ship is the maritime TT\&C (Telemetry, Track and Command) station to adapt to the missile and spacecraft experiment, and becomes an important part in our country's space flight control system at present.

During the process of navigation and anchoring, ship's attitude and shape will be influenced by different factors, such as stormy wave, tide and other environment factors. Meanwhile, position of the ship will also change. Compared with groundbased measurement data, the source of error is more complicated, and the trajectory data has more error components when it is got by ship's measurement system. Research shows that $[11,12,13]$, measurement accuracy of the ship is not only related to the measurement accuracy of ship-borne tracking equipment, but also to the accuracy of ship's shape, attitude and position. It means that in order to improve measurement accuracy, equipment's error caused by ship swing, deformation and position error should be analyzed and compensated. Especially for the random error of ship attitude measurement data, it will affect the accuracy of data processing due to coupling characteristic existing in the three attitude directions.

\section{B. Novel Method for Coupling Measurement Random Error}

For the measurement data of ship swing and ship deformation, the traditional method for suppressing random error is to make data-smooth in three direction angles separately. Because the three direction angles, i.e. yaw angle, pitching angle and rolling angle, of the ship swing or ship deformation have some coupling characteristic, and they are the rotation from one coordinate system to another, that is to say, it can map to the rotation group $S O(3)$, i.e. 3-dimensional proper orthogonal group. The rotation group $S O(3)$ is the $3-$ dimensional subspace in the 9-dimensional Euclid space. In order to make the most of the correlation, this paper improves the traditional wavelets to high dimensional data set by calculate the Lie-Algebra, and use this way to restrain the ship attitude random error.

The novel method that suitable to dealing with coupling random error is as follows:

(1)Calculate the transformation matrices,

$$
\begin{gathered}
B(\varphi)=\mathrm{K}_{\varphi} \Psi_{\varphi} \Theta_{\varphi} \\
B^{-1}(\varphi)=\mathrm{B}^{T}(\varphi)=\Theta_{\varphi}^{T} \Psi_{\varphi}^{T} \mathrm{~K}_{\varphi}^{T}
\end{gathered}
$$

with $\mathrm{K}_{\varphi}, \Psi_{\varphi}, \Theta_{\varphi}$ is the yaw angle, pitching angle and rolling angle, respectively, written as follows:

$$
\begin{aligned}
& \mathrm{K}_{\varphi}=\left(\begin{array}{ccc}
\cos k_{\varphi} & 0 & -\sin k_{\varphi} \\
0 & 1 & 0 \\
\sin k_{\varphi} & 0 & \cos k_{\varphi}
\end{array}\right), \Psi_{\varphi}=\left(\begin{array}{ccc}
\cos \psi_{\varphi} & -\sin \psi_{\varphi} & 0 \\
\sin \psi_{\varphi} & \cos \psi_{\varphi} & 0 \\
0 & 0 & 1
\end{array}\right), \\
& \Theta_{\varphi}=\left(\begin{array}{ccc}
1 & 0 & 0 \\
0 & \cos \theta_{\varphi} & -\sin \theta_{\varphi} \\
0 & \sin \theta_{\varphi} & \cos \theta_{\varphi}
\end{array}\right)
\end{aligned}
$$

(2)Multi-scale decomposition

To the matrices of attitude transform $\left\{B_{\varphi}\right\}$, we use the Log function to map the point in high dimensional data set to tangent vector in tangent space. Then interpolate the tangent vector, and use the Exp function to map the interpolation to the point in high dimensional data set.

Concretely, interpolate the half-integer point by using the sequence $B(\varphi) \in \mathrm{M}, \varphi \in \mathrm{Z}$, and choose a certain odd integer D. For example, if we want to calculate the imputation $\tilde{B}(1 / 2)$, we can use the data $B(l)$ at the $\mathrm{D}+1$ integer sites $l$ nearest to $1 / 2$. Letting $B(0)=B_{0}$ project the interpolating point to tangent space $_{T_{B_{0}}}(M)$ via

$$
\theta(l)=\log _{B_{0}}(B(l)), l=-(D-1) / 2, \ldots,(D+1) / 2
$$

Now, the points in tangent space belong to vector space, so they satisfy the linear operation. If we choose a base $\left\{e_{i}\right\}$ in ddimensional vector space, then $\theta(l)$ can be expressed as

$$
\theta(l)=\sum_{i=1}^{d} \tau_{i}(l) e_{i}
$$

Now, applying the algorithm to the true value sequence $\left\{\tau_{i}(l)\right\}$, we can get the interpolating point $\tilde{\tau}_{i}(1 / 2)$. Suppose that

$$
\tilde{\theta}(1 / 2)=\sum_{i=1}^{d} \tilde{\tau}_{i}(l) e_{i}
$$

Through the inverse mapping

$$
\begin{aligned}
& \qquad \tilde{B}(1 / 2)=\operatorname{Exp}_{B_{0}}(\tilde{\theta}(1 / 2)) \\
& \text { Calculate } \quad \text { in } \quad \text { order, } \\
& \text { get } \widetilde{B}\left((k+1 / 2) / 2^{j}\right), j=1,2, \ldots, d \text {. } \\
& \text { (3)Calculate the "wavelet" coefficients } \\
& \text { Let } \beta_{j, k} \equiv B\left(k / 2^{j}\right) \text {, then the "wavelet" coefficients can be }
\end{aligned}
$$
defined as follow:

$$
\alpha_{j, k}=\log _{\tilde{B}\left((k+1 / 2) / 2^{j}\right)} B\left((k+1 / 2) / 2^{j}\right)
$$

(4) Choose the threshold and do the "wavelet" denoising 
Since the "wavelet" coefficients are expressed in matrices actually, we need to calculate the matrices norm. Then the hard-threshold method can be used as follows: a norm threshold $\mathrm{T}$ is chosen firstly, and then the coefficient

whose norm is bigger than $\mathrm{T}$ is kept, the others are set to 0 . After this threshold eliminating, reconstitute method is used to process the new "wavelet” coefficients sequence.

The reconstitution algorithm mentioned above is listed as follows: when we get the sparse point sampling site $\left(\beta_{0, k}\right)$ and "wavelet" coefficients $\left(\left(\alpha_{j, k}\right) k \in Z\right)_{j \geq 0}$, we can reconstitute the signal

$$
\beta_{1,2 k+1}=\operatorname{Exp}_{\tilde{\beta}_{1,2 k+1}}\left(\alpha_{0, k}\right)
$$

$\tilde{\beta}_{2, k}$ can be calculated by $\beta_{1, k}$, then

$$
\beta_{2,2 k+1}=\operatorname{Exp}_{\tilde{\beta}_{2,2 k+1}}\left(2^{-1 / 2} \alpha_{1, k}\right)
$$

In this way, we can reconstitute the signal sequence.

\section{SIMULATION EXPERIMENT}

\section{A. Simulation Data}

In this section, taking the ship attitude measurement data as an example, we compare the proposed method with wavelet denoising method to show its validity and good performance.

As a matter of fact, we only have the measurement data of the ship attitude instead of the attitude true data, so we cannot compare the result. In the paper, we use simulation data to do the numeric experiment. In the process of experiment, in order to mirror the actual data's character as much as possible, we have some assumptions as follows:

i. The magnitude of the measurement data are conform to reality;

ii. The magnitude of the accuracy of the measurement data are conform to reality, that is to say, the standard deviation of the random error we add, meets a real;

iii. The ship attitude angle we simulate is correlative and coupling.

Under the above conditions, smooth the measurement data and the attitude angle as the theoretical attitude. As the same time, add the measurement random error to the smoothing data, and then generate the simulation data to do the numeric calculate and analysis.

\section{B. Result Analysis}

Now, we employ the proposed method to process the simulated data, and compare the result with traditional method. The results are shown in the Fig.1, Fig.2 and Fig.3.
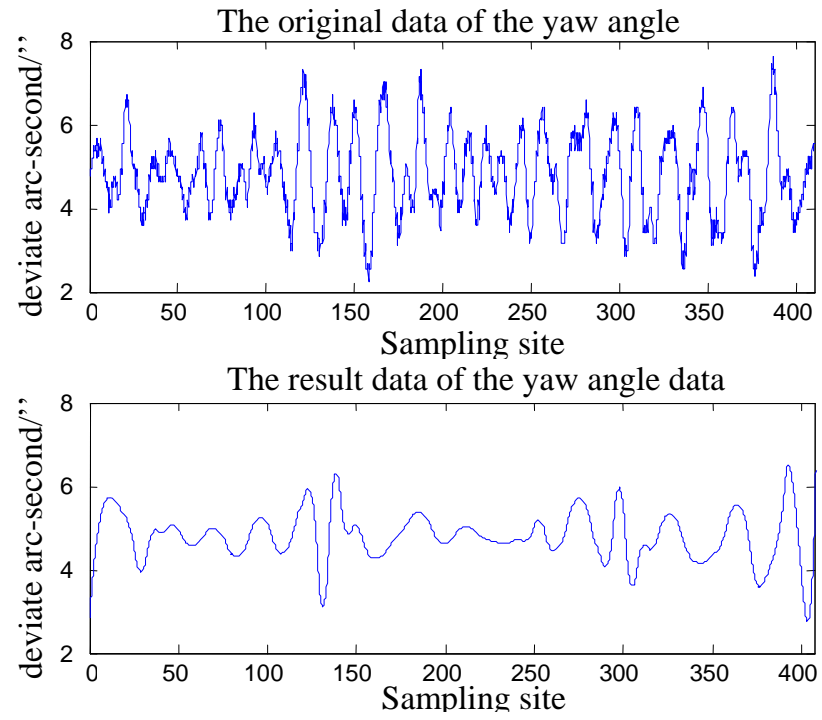

FIGURE I. THE RESULT OF THE YAW ANGLE
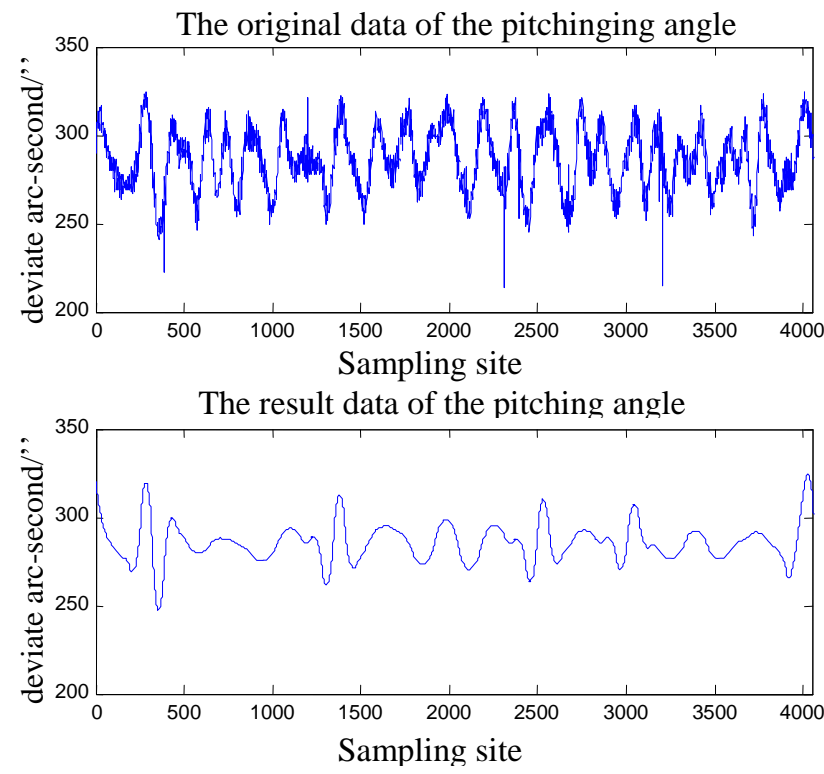

FIGURE II. THE RESULT OF THE PITCHING ANGLE 
The original data of the rolling angle

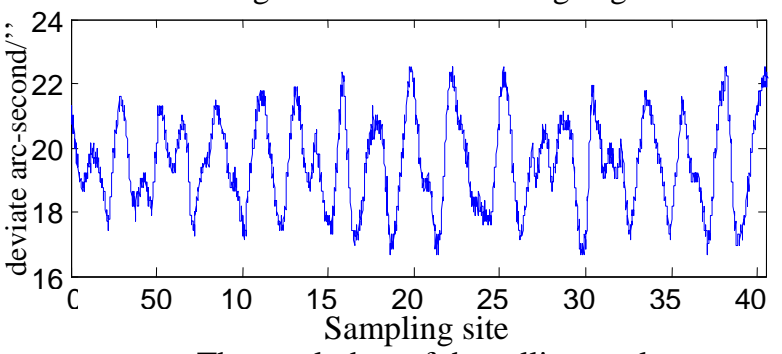

The result data of the rolling angle

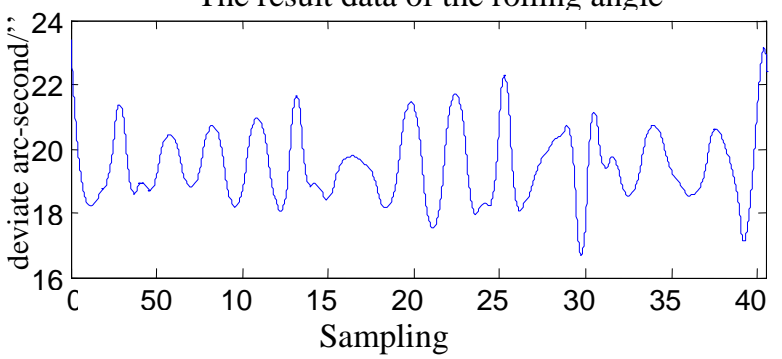

FIGURE III. THE RESULT OF THE ROLLING ANGLE

Meanwhile, we use the classical wavelet denoising way to deal with the three angles' random error. The result is listed in Table 1.

TABLE I. RESULT COMPARISON OF THE MULTISCALE ANALYSIS METHOD AND WAVELET DENOISING METHOD

\begin{tabular}{cccc}
\hline & $\begin{array}{c}\text { Wavelet } \\
\text { Method(arc- } \\
\text { second) }\end{array}$ & $\begin{array}{c}\text { Multiscale } \\
\text { Analysis } \\
\text { Method(arc- } \\
\text { second) }\end{array}$ & $\begin{array}{c}\text { Percentage } \\
\text { of } \\
\text { Improvement }\end{array}$ \\
\hline $\begin{array}{c}\text { Yaw } \\
\text { angle }\end{array}$ & 11.298 & 9.6033 & $15 \%$ \\
\hline $\begin{array}{c}\text { Pitchi } \\
\text { ng angle }\end{array}$ & 16.488 & 13.52 & $18 \%$ \\
\hline $\begin{array}{c}\text { Rolli } \\
\text { ng angle }\end{array}$ & 11.939 & 9.4319 & $21 \%$ \\
\hline
\end{tabular}

From the result, we can get the conclusion that the method proposed in this paper is better than the wavelet denoising method when dealing with the three angles. So the three angles are coupling.

\section{CONCLUSIONS}

For the coupled multi-measurement data, we propose a restrain method for coupled random error based on multiscale analysis on high dimensional data set in this paper. Combined with the classical wavelet decomposition and reconstitution theory, a "wavelet" transformation is given by calculating the point in high dimensional data set. Then, considered the coupling in the multi-measurement data, the structural characteristics of the high dimensional data set is used effectively. So the random error suppressing result is better. Finally, taken the three angles in ship attitude as the example, simulation is done. The result shows that the novel suppressing method can achieve better performance than the traditional wavelet denoising method.

\section{ACKNOWLEDGEMENTS}

This work was supported in part by Natural Science Foundation of China (NSFC) under Grant No. 61304119. The authors would like to thank reviewers and Professor H.Y. ZHOU in National University of Defence Technology for their constructive suggestions.

\section{REFERENCES}

[1] Bing Ye, Yetai Fei \& Benqiang Liao, The fuzzy degree of centrifugal and gross error detection. Chinese Journal of Mechanical Engineering. 19(1), pp.143-145, 2006.

[2] Mingfang Kong, Binzhen Chen, Bo Li \&Shanying Hu, Gross Error Identification for Steady State System. Journal of Chemical Engineering of Chinese Universities. 4(16), pp.430-435, 2002.

[3] Zhengming Wang, Dongyun Yi \&Xiaojun Duan, Measurement Data Modeling and Parameter Estimation, CRC press, Hunan, China, 2011.

[4] Stephane G. Mallat, A theory for multi-resolution signal decomposition: The wavelet representation. IEEE Transactions on Pattern Analysis and Machine Intelligence. 11(7), pp. 674-693, 1989.

[5] Dowine. T. R., Silverman B. W., The discrete multiple wavelet transform and thresholding methods. IEEE Transactions on signal processing, 46(9), pp. 2558-2561, 1998.

[6] Bui T D, Chen G., Translation-invariant denoising using multiwavelets. IEEE Transactions on signal processing, 46(12), pp. 3414-3429, 1998.

[7] N.E. Huang, Shen Z, \&S.R. Long et al., The empirical mode decomposition and the Hilbert spectrum for nonlinear and nonstationary time series analysis.ProcRsocLond, 454, pp. 56-78, 1998.

[8] Xiaolei Zhang, Yi Shen \& Yinchun Zhang, Novel fault diagnosis of satellite attitudecontrol system based on EMD. Journal of Huazhong University of Science and Technology (Natural Science Edition).8(37), pp. 204-206, 2009.

[9] Xiaogang Xu, Guanlei Xu \& Xiaotong Wang, Empirical Mode Decomposition and its Application. Chinese Journal of Electronics. 3(37), pp. 581-585, 2009.

[10] Inam Ur Rahman: Multiscale Representations for Manifold-Valued Data, Multi-scale Model Simulation. 4(4): 1201-1232, 2005

[11] Xiaoyong Li, Zhonghua Zhang, Tongshuang Zhang \& Deyong Kang, Characteristics analysis on spacecraft TT\&C ship swaying data. Journal of Chinese Inertial Technology. 3(14), pp.27, 2006.

[12] Yuyao Zhang, Min Li, Ping Yu \& Kui Shi, Research on angle diatortion for the measuring ship. Chinese Journal of Scientific Instrument. 6(27), pp.1505-1506, 1522, 2006.

[13] Huifen Li, Zhonghua Zhang \& Weikang Zhu, Correction Methods and Application Strategies for Hull Deformation of Space Instrumentation Ships. Journal of Spacecraft TT\&C Technology.3(29), pp.43,2010. 